\title{
An Investigation into Minimising Total Energy Consumption, Total Energy Cost and Total Tardiness Based on a Rolling Blackout Policy in a Job Shop
}

\author{
Ying Liu ${ }^{1}$, Niels Lohse ${ }^{1}$, Sanja Petrovic ${ }^{2}$, and Nabil Gindy ${ }^{3}$ \\ ${ }^{1}$ University of Nottingham, Department of Mechanical, \\ Material and Manufacturing Engineering, Nottingham, United Kingdom \\ \{epxy19, niels. lohse\} @nottingham.ac.uk \\ ${ }^{2}$ University of Nottingham, Department of Computer Science, Nottingham, United Kingdom \\ sanja.petrovic@nottingham.ac.uk \\ ${ }^{3}$ University of Nottingham Ningbo China, Division of Engineering, Ningbo, China \\ nabil.gindy@nottingham.edu.cn
}

\begin{abstract}
Manufacturing enterprises nowadays face the challenge of increasing energy price and emission reduction requirements. An approach to reduce energy cost and become environmental friendly is to incorporate energy consumption into consideration while making the scheduling plans. The research presented by this paper is set in a classical job shop circumstance, the model for the triple objectives problem that minimise total electricity cost, total electricity consumption and total tardiness when the Rolling Blackout policy is applied. A case study based on a $3 * 3$ job shop is presented to show how scheduling plans affect electricity consumption and its related cost, and to prove the feasibility of the model.
\end{abstract}

Keywords: Energy efficient production planning, sustainable manufacturing, job shop scheduling.

\section{Introduction}

Manufacturing industry is one of the most important energy consumers and carbon emitters in the world. For instance, every year in China, manufacturing generates at least $26 \%$ of the total carbon dioxide emission [1]. In order to reduce the carbon emission and balance the time-based unevenness of electricity demand, some countries, like China had promulgated corresponding electricity usage control policies and tariffs (EPTs), such as the Rolling Blackout policy for industry electricity supply, which means the government electricity will be cut off several days in every week resulting in manufacturing companies illegally starting their own diesel generators to maintain production. However, the private diesel electricity is more polluting and costly than the government supplied resource. Thus, the increasing price of energy and the current trend of sustainability have exerted new pressure on manufacturing enterprises, therefore they have to reduce energy consumption for cost saving and to become more environmentally friendly. As a result, employing operational methods to reduce the 
energy consumption and its related cost can be a feasible and effective approach for manufacturing enterprises [2]. The modelling method proposed in this paper can be applied to discrete event machining production system and may save significant amounts of energy and cost as well as keeping a good performance on classical scheduling objectives. The term "machining" will refer to processes such as milling, turning, drilling, and sawing [3]. In following content, the research problem will be raised after the research background and motivation; then the model will be presented, followed by a case study to demonstrate how scheduling plans affect the electricity consumption and its related cost in a job shop.

\section{Background and Motivation}

A considerable amount of research has been conducted in the area of sustainable machining. A detailed process model that can be used to determine the environmental impacts resulting from the machining of a particular part had been presented in [4]. The authors of [3] and [5] developed a system level research which not only includes energy requirement for material removal process itself, but also associated processes such as axis feed. The approach which breaks the total energy use of machining processes will be employed as the base for modelling electricity consumption of machine tools in this research.

Several operational methods, such as genetic algorithm, to minimise the electricity consumption and classical scheduling objectives on a single machine and parallel machines had been proposed in [6] and [7]. These methods are based upon the realization that in manufacturing environment, large quantities of energy are consumed by nonbottleneck machines as they lay idle. The Turn Off/On method developed in the above approach will be applied in this research. However, the applicable range of these works limits in single machine and parallel-machines circumstance. A modelling method for minimising energy consumption of manufacturing processes had been developed in [8], nevertheless, this research is based on the assumption that alternative routes with different energy consumption amounts exist for jobs in the manufacturing system. Therefore, the model is not applicable for workshops without, or having identical alternatives routes for jobs. What had been discussed above provides the motivation for this research from an academic aspect, i.e. that employing operational methods to reduce the electricity consumption in a typical job shop still has not been explored very well.

More importantly, from a practical aspect, the application of EPTs further complicate the aforementioned scheduling problem, since a scheduling plan that leads to reduction in electricity consumption does not necessarily lead to reduction in electricity cost in this situation. However, currently very little research focuses on this problem, even though it is important to deliver a trade-off between electricity consumption reduction and cost saving. Only [9] considered an instantaneous power limit in a case study. In this case the authors tried to use a discrete event simulation method to find a favourable solution. However, the solution quality could have been much improved if the intelligent search algorithms were applied.

Therefore, the new problem can be raised as: The Multi-objective Total Electricity Cost, Total Electricity Consumption and Total Tardiness Job Shop Scheduling 
problem based on Rolling Blackout policy (EC2T). The modelling method for this problem will be presented below.

\section{Models and Case Study}

In this section, models for EC2T will be defined. A case study of $3 * 3$ job shop will be presented to show how scheduling plans affect the objectives of Total Tardiness, Total Electricity Consumption and Total Electricity Cost.

\subsection{Job Shop Model}

Referring to [10] and [11], in the job shop scheduling problem, $J=\left\{J_{i}\right\}_{i=1}^{n}$, a finite set of $n$ jobs are to be processed on $M=\left\{M_{k}\right\}_{k=1}^{m}$, a finite set of $m$ machines following a predefined order.; $O_{i k}=\left\{O_{i k}^{l}\right\}_{l=1}^{u_{i}}$ is a finite set of $u_{i}$ ordered operations of $J_{i} ; O_{i k}^{l}$ is the $l$-th operation of $J_{i}$ processed on $M_{k}$ and it requires a processing time denoted $p_{i k}^{l}$. $S_{i k}^{l}$ indicates the time that $O_{i k}^{l}$ begins to be processed on $M_{k}$, while $C_{i k}^{l}$ is the corresponding completion time of that process. $Y_{i i^{\prime} k}^{l l^{\prime}}$ is a decision variable that $Y_{i i^{\prime} k}^{l l^{\prime}}=1$ if $O_{i k}^{l}$ precedes $O_{i^{\prime} k}^{l^{\prime}}$ on $M_{k}, 0$ otherwise. Each $J_{i}$ has a release time into the system $r_{i}$ and a due date $d_{i}$. $w_{i}$ is the weight associated with $J_{i}$.

Constraints:

$$
\begin{gathered}
S_{i k}^{l} \geq r_{i} \\
C_{i k}^{l+1}-C_{i k^{\prime}}^{l} \geq p_{i k}^{l+1} ; \quad S_{i k}^{l+1}-C_{i k^{\prime}}^{l} \geq 0 \\
C_{i^{\prime} k}^{l^{\prime}}-C_{i k}^{l} \geq p_{i^{\prime} k}^{l^{\prime}} Y_{i i^{\prime}}^{l l^{\prime}}=1
\end{gathered}
$$

Where

$Y_{i i^{\prime} k}^{l l^{\prime}} \in\{0,1\} ; S_{i k}^{l} \geq 0 ; C_{i k}^{l} \geq 0 ; C_{i k^{\prime}}^{l} \geq 0 ; k \neq k^{\prime} ; i \neq i^{\prime} ; 1 \leq l \leq u_{i}$

$\forall J_{i}, J_{i^{\prime}} \in J ; \forall O_{i k}^{l}, O_{i k}^{l+1}, O_{i k^{\prime}}^{l} \in O_{i k} ; \forall O_{i^{\prime} k}^{l^{\prime}} \in O_{i^{\prime} k} ; \forall M_{k}, M_{k^{\prime}} \in M$

Constraint (1) makes sure that the starting time of any job must greater than its release time. Constraint (2) ensures that the precedence relationships between the operations of a job are not violated, i.e. the $O_{i k}^{l+1}$ is not started before the $O_{i k^{\prime}}^{l}$ had been completed, and no job can be processed by more than one machine at a time. Constraint (3) takes care of the requirement that no machine can process more than one operation at a time, i.e. no pre-emption is allowed. A schedule $s$ that complies with constraints (1) to (3) is said to be a feasible schedule. $S$ is a finite set of all feasible schedules that $s \in S$. Given a feasible schedule $s$, let $C_{i}(s)$ indicate the completion time of $J_{i}$ in schedule $s$. The tardiness of $J_{i}$ can be denoted as $T_{i}(s)=\max \left\{0, C_{i}(s)-d_{i}\right\}$. The objective is to minimise the total weighted tardiness of all jobs.

$$
\operatorname{minimise}\left(\sum_{i=1}^{n} w_{i} \times T_{i}(s)\right)
$$




\subsection{Electricity Consumption Model}

Based on existing research work on environmental analysis of machining [5], [12], [13], [14], the simplified power input model of $M_{k}$ when it is working on $O_{i k}^{l}$ is shown in Fig. 1.

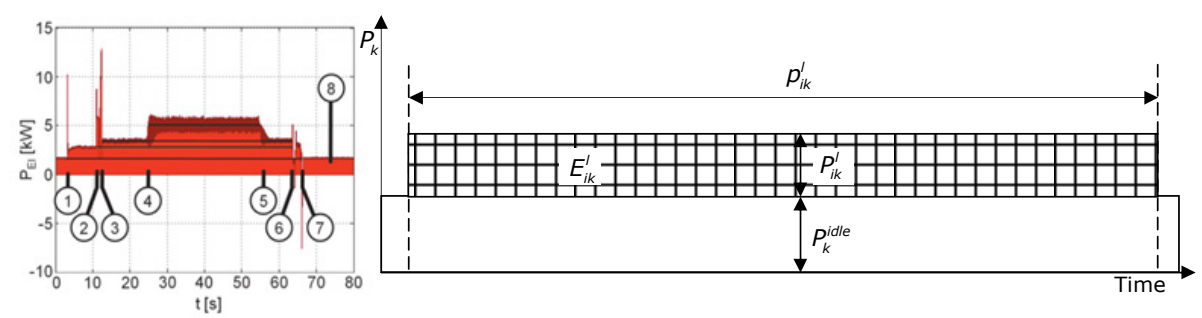

Fig. 1. Actual power input at machine tool main connection over time and its simplified model when it is working on one operation (based on [12])

$P_{k}^{i d l e}$ refers to the idle power of $M_{k}, P_{i k}^{\text {lruntime }}$ and $E_{i k}^{\text {lruntime }}$ represent the power and energy consumed by $M_{k}$ when it executes the runtime operations for processing $O_{i k}^{l}$ that $E_{i k}^{\text {lruntime }}=P_{i k}^{\text {lruntime }} \times p_{i k}^{l} . p_{i k}^{l}$ is defined as the time interval between coolant switching on and off. $P_{i k}^{\text {lcutting }}$ and $E_{i k}^{\text {lcutting }}$ are the power and energy consume by $M_{k}$ when it actually executes cutting for $O_{i k}^{l}, E_{i k}^{\text {lcutting }}=$ $P_{i k}^{\text {lcutting }} \times t_{i k}^{\text {lcutting }} . t_{i k}^{\text {lcutting }}$ is the corresponding cutting time. $P_{k}=P_{k}^{\text {idle }}+$ $P_{i k}^{\text {lruntime }}+P_{i k}^{\text {lcutting }}$. $E_{i k}^{\text {lruntime }}, E_{i k}^{\text {lcutting }}$ and $P_{k}^{\text {idle }}$ can be seen as constants when both of the product's and machine tool's characteristics are known. Thus, define $E_{i k}^{l}$ as energy consumed by runtime operations and cutting of $O_{i k}^{l}$ on $M_{k}$, that $E_{i k}^{l}=E_{i k}^{\text {lruntime }}+E_{i k}^{\text {lcutting }}$. $E_{i k}^{l}$ can be seen as a constant. To simplify the power input model, it is supposed that all the runtime operations and the actual cutting share the same starting and ending time. Therefore, define $P_{i k}^{l}$ as the average power input of $M_{k}$ during $p_{i k}^{l}, P_{i k}^{l}=E_{i k}^{l} / p_{i k}^{l}, P_{k}=P_{k}^{i d l e}+P_{i k}^{l}$. This model will simplify the calculation of total electricity consumption and electricity cost of the job shop, as well as guarantee the necessary accuracy for EC2T problem. Based on the model discussed above, it is easy to see that $E_{i k}^{l}$ is the processing related energy consumption, and $\sum E_{i k}^{l}$ will not be affected by different scheduling plans. Thus, the objective to reduce total electricity consumption of a job shop can be converted to reduce total nonprocessing electricity consumption which includes idle and Turn Off/On electricity consumption of machine tools [7].The objective function can be set as:

$$
\operatorname{mimise}\left(\sum_{k=1}^{m} T E M_{k}^{n p}\right)
$$

$T E M_{k}^{n p}$ is the non-processing electricity consumption of $M_{k} . M_{k}^{\prime}=\left\{m_{k}^{r}\right\}_{r=1}^{\sum_{i=1}^{n} \Sigma_{l=1}^{u_{i}} \gamma_{i k}^{l}}$ is a finite set of operations processed on $M_{k} \cdot \gamma_{i k}^{l}$ is a decision variable that $\gamma_{i k}^{l}=1$ if the $l$-th operation of $J_{i}$ processed on $M_{k}, 0$ otherwise. $S_{k}^{r}$ and $C_{k}^{r}$ respectively 
indicate the start and completion time of $m_{k}^{r}$ on $M_{k}$. A schedule $s$ can be graphically expressed as a Gantt Chart, the calculation of the total non-processing electricity consumption will be based on it. Fig. 2 is an example for the calculation. $O_{i_{1} k}^{l_{1}}, O_{i_{2} k}^{l_{2}}$, $O_{i_{3} k}^{l_{3}}$, and $O_{i_{4} k}^{l_{4}}$ are processed by $M_{k}$. The Turn Off/On method suggested by [6] is allowed, then:

$$
T E M_{k}^{n p}=P_{k}^{\text {idle }} \times\left[\max \left(C_{k}^{r}\right)-\sum_{r}\left(C_{k}^{r}-S_{k}^{r}\right)-\sum_{r}\left(S_{k}^{r+1}-C_{k}^{r}\right) \times Z_{k}^{r}\right]+E_{k}^{\text {turn }} \times \sum_{r} Z_{k}^{r}
$$

According to [6], $E_{k}^{\text {turn }}$ is the the energy consumed by Turn Off/On; $B_{k}$ is the break-even duration of machine for which Turn Off/On is economically justifiable instead of running the machine idle, $B_{k}=E_{k}^{\text {turn }} / P_{k}^{\text {idle }} . t_{k}^{\text {OFF }}$ is the time required to turn off then turn on $M_{k} ; Z_{k}^{r}$ is a decision variable that $Z_{k}^{r}=1$ if $S_{k}^{r+1}-C_{k}^{r} \geq$ $\max \left(B_{k}, t_{k}^{O F F}\right), 0$ otherwise.

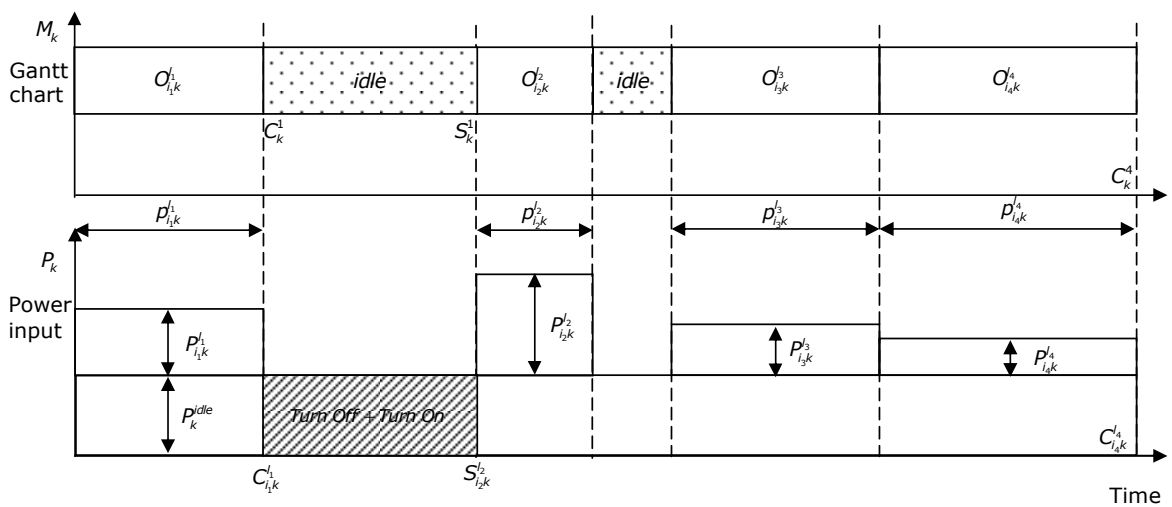

Fig. 2. Gantt chart of $M_{k}$ and its corresponding power profile

\subsection{Electricity Cost Model (Based on the Rolling Blackout Policy)}

The objective function for electricity cost of a job shop is:

$$
\begin{gathered}
\operatorname{minimise}(\operatorname{TEC}(s)) \\
\operatorname{TEC}=\sum_{k=1}^{m} \operatorname{TEC}_{k}(s) \\
T E C_{k}(s)=p^{e} \times \int_{0}^{\max \left(C_{k}^{r}\right)} P_{k} \quad p^{e}= \begin{cases}\beta_{1}, & t \in\left[(n-1) T,(n-1) T+t_{s}\right) \\
\beta_{2}, & t \in\left((n-1) T+t_{s}, n T\right]\end{cases}
\end{gathered}
$$

As seen in Fig. 3, $T E C(s)$ and $T E C_{k}(s)$ respectively refer to the total electricity cost of the job shop and $M_{k}$ in a feasible schedule $s ; p^{e}$ represents the electricity price that $p^{e}=\beta_{1}$ Pounds $/ K w h$ if it is government electricity supply, while $p^{e}=\beta_{2}$ Pounds $/ K w h$ if it is private diesel electricity supply. $T$ denotes the cycle period of Rolling Blackout policy. $t_{s}$ has separated $T$ to $\Delta t_{s}$ and $\Delta t_{o}$ that indicate the period of government and private electricity supply respectively. In this model, $n$ is the natural numbers starting from $1 ; t$ indicates the time. Referring to [15], the objective function of EC2T can be expressed as: 


$$
\begin{gathered}
\text { minimise } F(s)=\left(f_{1}(s), f_{2}(s), f_{3}(s)\right) s \in S \\
f_{1}(s)=\sum_{i=1}^{n} w_{i} \times T_{i}(s) ; f_{2}(s)=\sum_{k=1}^{m} T E M_{k}^{n p} ; f_{3}(s)=T E C(s)
\end{gathered}
$$

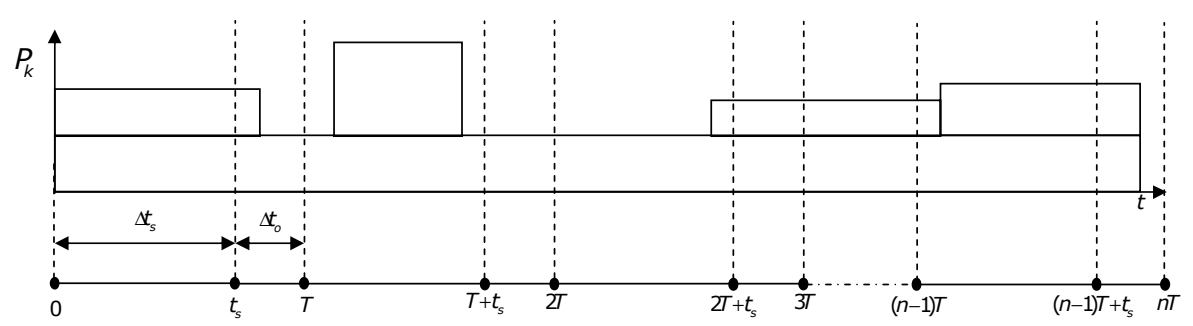

Fig. 3. The timeline for TOU tariff and the power input profile of machine tool

\subsection{Case Study}

Parameters of the $3 * 3$ job shop [16] is given in Table 1 , numbers in the brackets are the values of $p_{i k}^{l}$. The values for the parameters are assumed, based on experiments of [17] in Table 2. Fig. 4 shows a comparison between two feasible schedules. The reasonable Turn Off/On plans for each of them are developed. The values of the three objectives of the two plans are shown in Table 3 .

Table 1. The $3 * 3$ job shop

\begin{tabular}{|l|c|c|c|l|l|}
\hline$O_{i k}^{l}$ & $O_{i k}^{1}$ & $O_{i k}^{2}$ & $O_{i k}^{3}$ & Release time & Due date \\
\hline$J_{i}$ & $M_{1}(2)$ & $M_{2}(2)$ & $M_{3}(3)$ & 0 & The $10^{\text {th }}$ time unit \\
\hline$J_{2}$ & $M_{3}(3)$ & $M_{2}(1)$ & $M_{1}(4)$ & 0 & The $10^{\text {th }}$ time unit \\
\hline$J_{3}$ & $M_{2}(1)$ & $M_{1}(3)$ & $M_{3}(2)$ & 0 & The $10^{\text {th }}$ time unit \\
\hline
\end{tabular}

Table 2. Values for other parameters

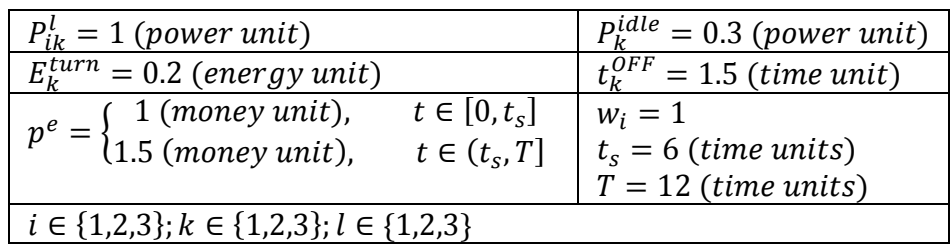

Table 3. Values of three objectives for two scheduling plans

\begin{tabular}{|c|c|c|c|}
\hline$f(s) s$ & $\begin{array}{l}\text { Total weighted } \\
\text { tardiness }\end{array}$ & $\begin{array}{l}\text { Total non-processing } \\
\text { electricity consumption }\end{array}$ & $\begin{array}{l}\text { Total electricity } \\
\text { cost }\end{array}$ \\
\hline$s_{1}$ & 0 & 0.5 energy unit & 32.35 money units \\
\hline$s_{2}$ & 1 & 0.4 energy unit & 32.90 money units \\
\hline
\end{tabular}

$s_{1}$ outperforms on minimising total tardiness, while $s_{2}$ outperforms on minimising total non-processing electricity consumption. However, when applying the Rolling Blackout policy, the comparison between the two schedules on minimising total 
electricity cost demonstrates that scheduling plan $\left(s_{2}\right)$ that reduces electricity consumption does not necessarily reduce electricity cost. This simple case could demonstrate the feasibility of the aforementioned model. The complexity of the problem will increase along with the increasing numbers of jobs and machines, various energy characteristic of machine tools and the application of different electricity control policies and tariffs.

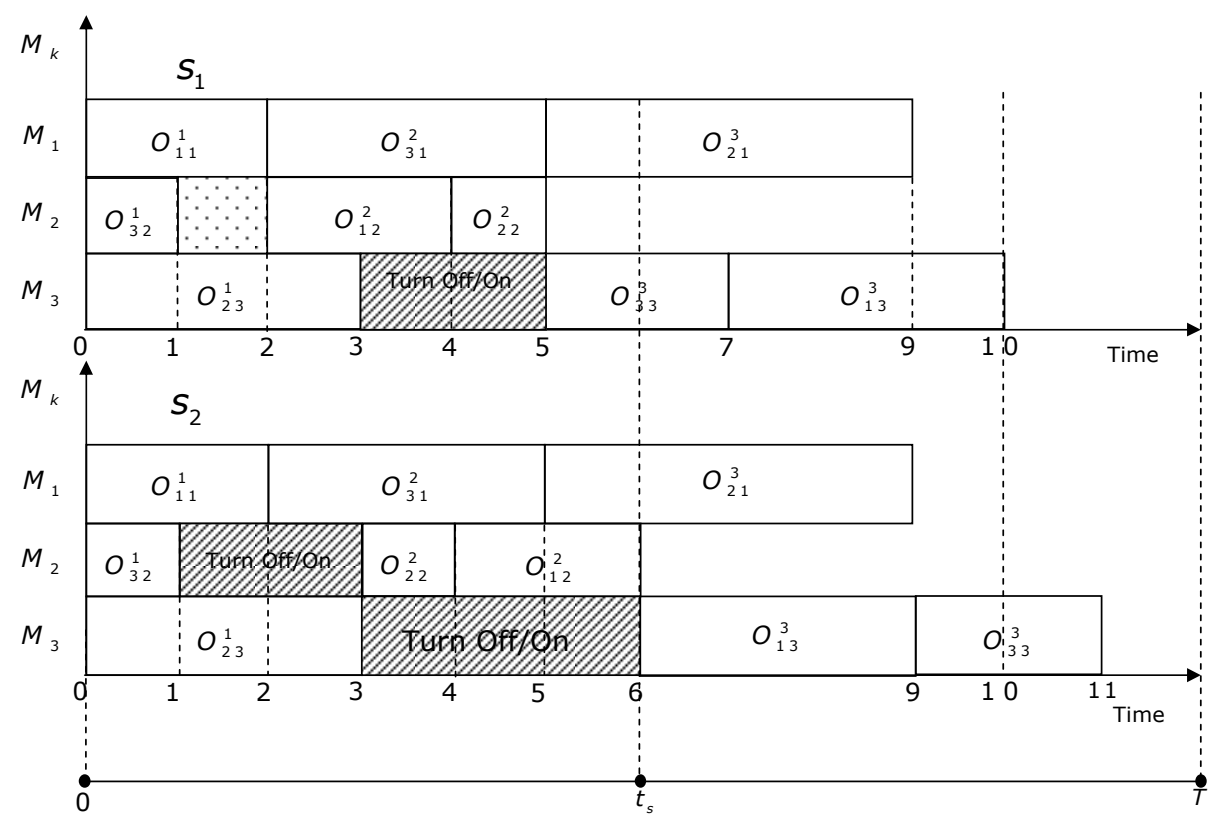

Fig. 4. Comparison between two feasible schedules on total electricity consumption and total tardiness

\section{Conclusion and Future Work}

Reducing electricity consumption and its related cost as well as keeping good performance in classical job shop scheduling objectives is a difficult problem that can take a large amount of time if optimally solved. The model for EC2T had been developed in this paper. A case study had been presented to show how scheduling plans affect the objectives of total tardiness, total non-processing electricity consumption and total electricity cost. Obviously, the differences in electricity consumption and its related cost among different scheduling plans will increase along with the increasing units in each job. This provides new insight for managers that optimisation in scheduling is not only a factory performance improvement approach, but also a route that leads to sustainability. In future work, more complicated job shop instance will be studied based on the aforementioned model. The Non-dominated Sorting Genetic Algorithm (NSGA-II) had been selected as the problem solving approach. For more information refer to [18]. In addition, various situations about job arrival patterns will also be taken into consideration in the future work. 


\section{References}

1. Tang, D., Li, L., Du, K.: On the Developmental Path of Chinese Manufac-turing Industry Based on Resource Restraint. Jiangsu Social Sciences 4, 51-58 (2006)

2. Mouzon, G., Yildirim, M.B.: A framework to minimize total energy con-sumption and total tardiness on a single machine. In: Proceedings of 4th Annual GRASP Symposium, vol. 1(2), pp. 105-116 (2008)

3. Dahmus, J.B., Gutowski, T.G.: An environmental analysis of machining. In: Proceedings of ASME International Mechanical Engineering Congress and RD\&D Expo 2004, pp. 1-10 (2004)

4. Munoz, A.A., Sheng, P.: An analytical approach for determining the envi-ronmental impact of machining processes. Journal of Materials Processing Technology 53, 736-758 (1995)

5. Kordonowy, D.: A power assessment of machining tools. Massachusetts Institue of Technology (2003)

6. Mouzon, G., Yildirim, M.B., Twomey, J.: Operational methods for mini-mization of energy consumption of manufacturing equipment. Interna-tional Journal of Production Research 45(18-19), 4247-4271 (2007)

7. Mouzon, G.: Operational methods and models for minimization of energy consumption in a manufacturing environment. Wichita State University (2008)

8. He, Y., Liu, B., Zhang, X., Gao, H., Liu, X.: A modeling method of task-oriented energy consumption for machining manufacturing system. Journal of Cleaner Production 23(1), 167-174 (2012)

9. Herrmann, C., Thiede, S.: Process chain simulation to foster energy effi-ciency in manufacturing. CIRP Journal of Manufacturing Science and Technology 1, 221-229 (2009)

10. Özgüven, C., Özbakır, L., Yavuz, Y.: Mathematical models for job-shop scheduling problems with routing and process plan flexibility. Applied Mathematical Modelling 34(6), 1539-1548 (2010)

11. Antonio, J., Petrovic, V.S.: A new dispatching rule based genetic algorithm for the multiobjective job shop problem. Journal of Heuristics, 771-793 (2010)

12. Dietmair, A., Verl, A.: Energy Consumption Forecasting and Optimisation for Tool Machines. Energy, 63-67 (2009)

13. Diaz, N., et al.: Machine Tool Design and Operation Strategies for Green Manufacturing. In: Proceedings of 4th CIRP Internatinal Conference on High Performance Cutting, pp. 1-6 (2010)

14. Avram, I.O.: Machine Tool Use Phase: Modeling and Analysis with Environmental Considerations," ÉCOLE POLYTECHNIQUE FÉDÉRALE DE LAUSANNE (2010)

15. Vázquez-Rodríguez, J.A., Petrovic, S.: A new dispatching rule based genetic algorithm for the multi-objective job shop problem. Journal of Heuristics 16(6), 771-793 (2009)

16. Liu, M., Wu, C.: Intelligent Optimization Scheduling Algorithms for Manufacturing Process and Their Applications, p. 334. National Defense Industry Press (2008)

17. Lv, J.X., Tang, R.Z., Jia, S.: Research on energy consumption modeling of CNC machine tool for non-cutting operations, no. 51175464 (2012)

18. Deb, K., Pratap, A., Agarwal, S., Meyarivan, T.: A Fast and Elitist Multiobjective Genetic Algorithm: NSGA-II 6(2), 182-197 (2002) 